\title{
Central Cholinergic Descending Pathway to the Dorsal Motor Nucleus of the Vagus in Regulation of Gastric Functions
}

\author{
Yasunobu OKUMA and Yoshitsugu OSUMI \\ Department of Pharmacology, Kochi Medical School, Nankoku, Kochi 781-51, Japan
}

Accepted April 10, 1986

\begin{abstract}
A possible role of the cholinergic mechanism within the dorsal motor nucleus of the vagus (NDV) in the regulation of gastric functions was examined in urethane anesthetized rats. Pretreatment with atropine (1-5 nmole), intracerebroventricularly inhibited the increase in both gastric acid output and mucosal blood flow (MBF), as induced by electrical stimulation of the lateral hypothalamic area (LHA). Bethanechol, microinjected into the dorsal vagal complex (the NDV. the nucleus tractus solitarius and area postrema) induced dose-dependent (5-30 nmole) increases in gastric acid output and MBF, while nicotine was without effect. Pretreatment with atropine 3 nmole microinjected into the dorsal vagal complex completely blocked the increases induced by electrical stimulation of the LHA. Furthermore, the increases in these gastric parameters induced by the administration of 2-deoxy-D-glucose (2-DG) were also significantly inhibited by atropine microinjected into the dorsal vagal complex. These results suggest that neurotransmission of the central descending pathway to the NDV in excitatory regulation of gastric functions is probably mediated through cholinergic muscarinic receptors.
\end{abstract}

The role of the central nervous system in regulation of gastric function has been extensively examined at the level of the hypothalamus (1-4) and the NDV $(5,6)$. We have already reported that there exists a central noradrenergic inhibitory mechanism in regulation of gastric functions $(7,8)$, and the sites of action were probably the NDV (9) and/or the LHA (7). On the other hand, central cholinergic mechanisms are thought to be excitatory in regulation of gastric functions. Intraventricular or intracarotid infusion of acetylcholine increased gastric secretion $(10,11)$, and cholinergic agonists such as pilocarpine and methacholine enhanced gastric secretion when applied into the inferior cerebellar peduncle or supraoptic nucleus $(12,13)$. We have recently found that acetylcholine and bethanechol applied into the LHA enhanced gastric acid secretion (7).

The LHA is defined as the so-called "feeding center", and activation of this LHA neuron in rats results in a significant increase in the volume of gastric secretion and acid output (2). This effect can be explained by activation of motor pathways originating from the LHA and descending to the brain stem NDV (6). Furthermore, increases in gastric acid secretion induced by excitation of the central nervous system such as by administration of 2-DG $(14,15)$, intraventricular administration of TRH (16) and CCK-8 (17) appear to produce their effects through a vagally mediated mechanism. The NDV contains cell bodies of the efferent cholinergic component of the vagus nerves. However, mechanisms of transmission at the level of the NDV of the LHA-NDV descending pathways remain obscure.

Whether or not the cholinergic component is involved in these descending pathways for central excitation of gastric functions was examined in rats anesthetized with urethane, and the observations are described herein.

\section{Materials and Methods}

Male Wistar rats weighing $220-250 \mathrm{~g}$ were maintained in a room at $22-24^{\circ} \mathrm{C}$ under a constant day-night rhythm for 7-10 days 
and given food (laboratory chow, CA-1, Japan Clea Co.) and tap water, ad libitum. Prior to each experiment, all food but not water was withheld for $16 \mathrm{hr}$. Under urethane anesthesia (1 g/ $\mathrm{kg}$, i.p.), the femoral vein and femoral artery were cannulated. The abdomen was opened by a transverse incision and a round-tip cannula $(5 \mathrm{~cm}$ long. $0.5 \mathrm{~cm}$ outer diameter) connected to a polyethylene tube was inserted into the stomach via an incision into the duodenum $(1 \mathrm{~cm}$ distal from the pyloric sphincter). The tip of the cannula lay just above the pyloric sphincter and was held in place by two ligatures around the duodenum, one at the point of incision and the other close to the pylorus, as described by Main and Whittle (18). To remove the solid contents, the stomach was flushed with saline, taking care to avoid distention. After repeated washings, two $\mathrm{ml}$ of solution prewarmed to $38^{\circ} \mathrm{C}$ was placed in the stomach at the beginning of each $15 \mathrm{~min}$ collection period. The composition of this solution was a 1:5 (v/v) mixture of glycine and mannitol adjusted to $300 \operatorname{mos} \mathrm{M}$ and $\mathrm{pH}$ 3.5 by addition of $0.1 \mathrm{~N} \mathrm{HCl}$, according to the method of Blair et al. (19).

Acid output was determined by titration of gastric samples to $\mathrm{pH} 7.0$ with $0.01 \mathrm{~N} \mathrm{NaOH}$. using a $\mathrm{pH}$ meter. MBF was measured by the aminopyrine clearance technique developed by Jacobson et al. (20) as based on the $\mathrm{pH}$ partition theory of Shore et al. (21). Thirty min after the priming dose of aminopyrine $30 \mathrm{mg} / \mathrm{kg}$, i.p., $6.6 \mathrm{mg} / \mathrm{kg} / \mathrm{hr}$ infusion through the femoral vein was started and was continued throughout the experiment to maintain a constant blood level of aminopyrine.

The animal was placed in a stereotaxic instrument. Forty-five min were allowed for stabilization of acid and aminopyrine contents in the gastric juice after the onset of aminopyrine infusion. To ensure that the rats remained in good condition, the total volume of blood samples was kept to a minimum. Samples of arterial blood $(0.5 \mathrm{ml})$ were collected via a cannula inserted into the femoral artery, once $60 \mathrm{~min}$ after the onset of aminopyrine infusion and again immediately after the end of the experiment. The plasma level of aminopyrine at each 15 min interval during the experimental period was estimated by interpolation between two measured points and was paired with each aminopyrine determination in the gastric juice. The contents of aminopyrine in the plasma and gastric juice sample were assayed by the method of Brodie and Axelrod (22). The gastric MBF was calculated from these measurements (8).

To increase the basal levels of gastric acid output and MBF, in one series of experiments, the LHA was electrically stimulated. In another series of experiments, 2-DG $60 \mathrm{mg} /$ $\mathrm{kg}$ was administered intravenously.

Bipolar stainless steel electrodes $(0.1 \mathrm{~mm}$ diameter) were used for electrical stimulation of the LHA (AP: 5.0. L: 1.5, H: 2.0), following the brain atlas by König and Klippel (23). For electrical stimulation of the LHA, square pulses of $0.5 \mathrm{~mA}, 2 \mathrm{msec}, 10$ cycles/sec were applied for 10 min by means of an electronic stimulator (Model 3201. Nihon Kohden Ltd., Japan). By repetitive electrical stimulations, the second stimulation was performed $75 \mathrm{~min}$ after the first one. Pretreatment with atropine was given $15 \mathrm{~min}$ prior to the second stimulation. The results were expressed as the net increases in gastric parameters by the stimulations. The $S_{1}$ and $S_{2}$ represent the values at the first and second stimulations, respectively, and the effect of atropine was evaluated as the ratio $S_{2} / S_{1}$ (Fig. 4 ).

Drugs were dissolved in saline or in artificial cerebrospinal fluid. The composition of the artificial cerebrospinal fluid, as described by Falcon et al. (24), was $7.3 \mathrm{mg}$ $\mathrm{NaCl}, 1.9 \mathrm{mg} \mathrm{NaHCO}, 0.3 \mathrm{mg} \mathrm{MgSO}_{4}$, $0.2 \mathrm{mg} \mathrm{CaCl}, 0.2 \mathrm{mg} \mathrm{NaH} \mathrm{PO}_{4}$ and $0.8 \mathrm{mg}$ glucose in $1 \mathrm{ml}$ of deionized water; then the solutions containing test substances were applied into the lateral cerebral ventricle (AP: 7.5. L: 1.1, H: $5.0 \mathrm{~mm}$ from the cortical surface) in a volume of $10 \mu$ through a stainless steel micropipette $(0.35 \mathrm{~mm}$ outer diameter). For the application of test substances into the dorsal vagal complex, a glass micropipette (70 $/ \mathrm{m}$ outer diameter) was placed in an area just above the NDV within the dorsal vagal complex $(\mathrm{L}: 0.5, \mathrm{H}$ : 0.6, AP: -6.0 ). Then, $0.5 \mu l$ of solution containing test substances was applied through this micropipette.

in the experiments using 2-DG, micro- 


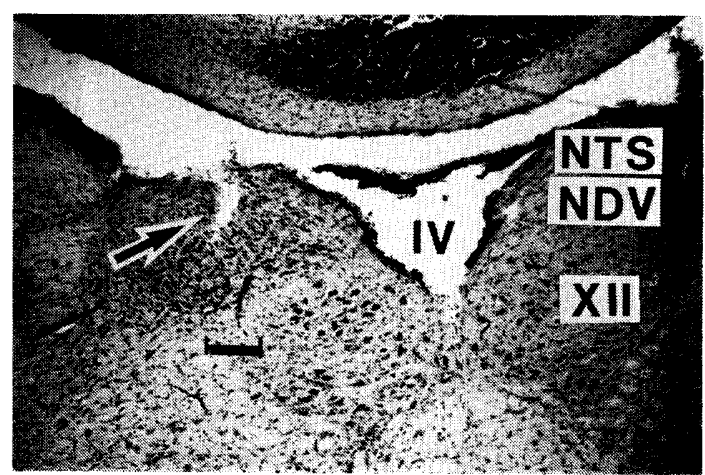

Fig. 1. Representative illustration of the microinjection site. Histological sections were stained with cresyl-violet. Horizontal bar: $0.1 \mathrm{~mm}$. Arrow: locations of the tips of micropipette. NDV: dorsal motor rucleus of the vagus, NTS: nucleus tractus solitarius, XII: nucleus of the hypoglossal nerve. IV: fourth ventricle.

injection of atropine into the dorsal vagal complex was performed bilaterally $(0.25$ nmole $\times 2$ or 1.5 nmole $\times 2$ ).

In experiments with $2-D G$, administration of artificial cerebrospinal fluid alone into the dorsal vagal complex induced decreases in gastric parameters. Therefore, we used saline as a vehicle for microinjections in the experiment using 2-DG as well as in the first experiment with bethanechol. After the experiment, the brain was removed, fixed in $10 \%$ formalin, and the frozen sections cut at $30 \mu \mathrm{m}$ were stained with cresyl violet for microscopic study of the location of both the electrical stimulation and microinjection sites. The histological picture of the microinjection site of test substances within the dorsal vagal complex is representatively illustrated in Fig. 1. Results were expressed as the mean \pm S.E. Statistical significance was compared with the corresponding values of control rats using Student's t-test for unpaired comparisons.

\section{Results}

The mean basal gastric acid output and MBF obtained from rats under urethane anesthesia were $5.04 \pm 0.67 \mu \mathrm{Eq} / 15 \mathrm{~min}$ $(n=48)$ and $1.58 \pm 0.11 \mathrm{ml} / 15 \mathrm{~min}(n=48)$.

Effects of cholinergic agonists microinjected into the dorsal vagal complex on gastric acid output and MBF: Microinjection of saline into the dorsal vagal complex unilaterally slightly increased gastric acid output and MBF. Similar results were reported by Kadekaro et al. (5).

Bethanechol microinjected into the dorsal vagal complex induced a dose-dependent (5-30 nmole) increase in both gastric acid output and MBF. On the other hand, 3 nmole nicotine did not significantly affect the levels of these gastric parameters, as reported (25). and this alkaloid was without effect even in a dose of 30 nmole (Fig. 2). From these results, the site of action of bethanechol is probably within the dorsal vagal complex; however, a possibility that part of the bethanechol leaked into other brain regions or leaked out of the brain to directly affect gastric functions in the stomach still remains unresolved. Then, it was confirmed that 30 nmole bethanechol intraventricularly and intravenously administered, did not significantly affect gastric acid secretion (data not shown).

Effects of intraventricularly applied atropine on increases in gastric acid output and MBF induced by stimulation of the LHA:

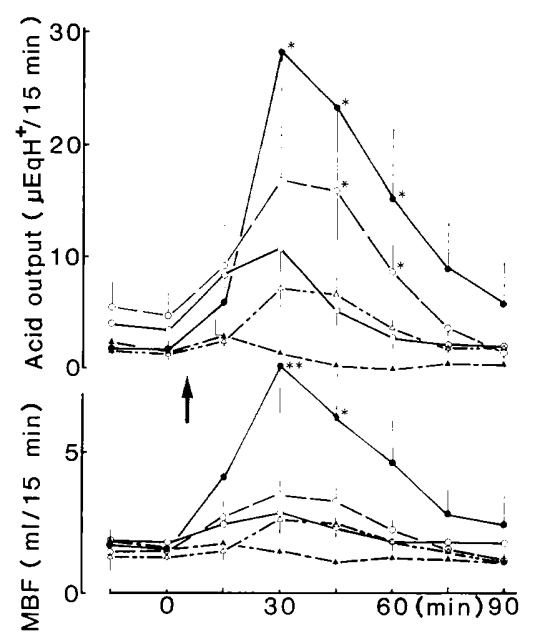

Fig. 2. Effects of bethanechol and nicotine microinjected into the dorsal vagal complex on the basal gastric acid output and MBF. $\uparrow$ : Administration of test substances into the dorsal vagal complex. $\bigcirc-O$ : saline $(n=10), \bigcirc--O: 5$ nmole bethanechol $(n=10)$, 30 nmole bethanechol $(n=7), \triangle \cdots-\triangle: 3$ nmole nicotine $(n=7)$, $\mathbf{\Delta}--\mathbf{\Delta}: 30$ nmole nicotine $(n=3) .{ }^{*} P<0.05$, ${ }^{*} P<0.01$ (statistically significant difference from the control with saline). 


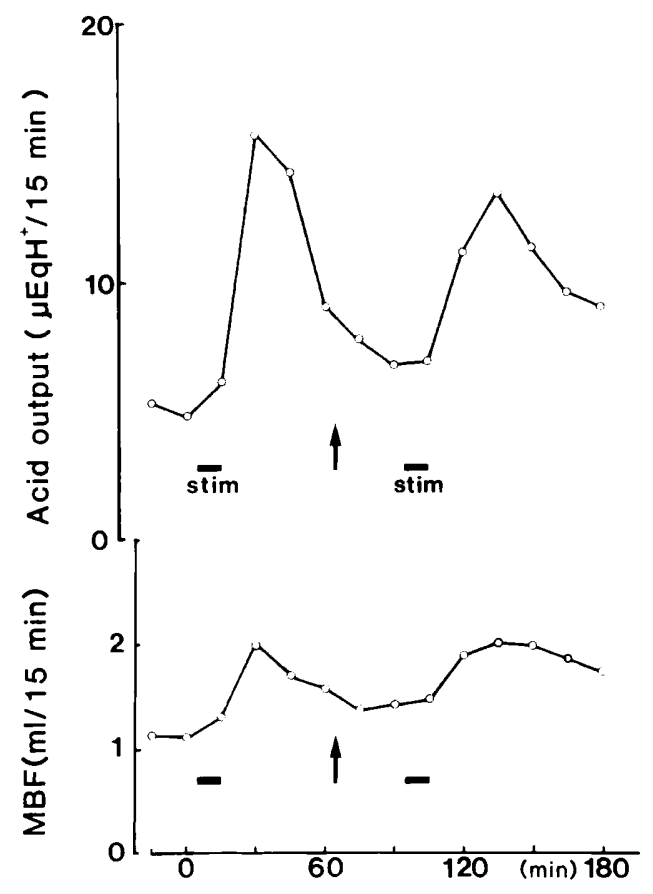

Fig. 3. Repeated electrical stimulation of the LHA on the gastric acid output and MBF in a control rat. Vehicle, artificial cerebrospinal fluid, was microinjected into the ipsilateral dorsal vagal complex 15 min prior to the second electrical stimulation. Stim: electrical stimulation (0.5 mA, 2 msec, 10 cycles/ sec, for $10 \mathrm{~min}$ ).

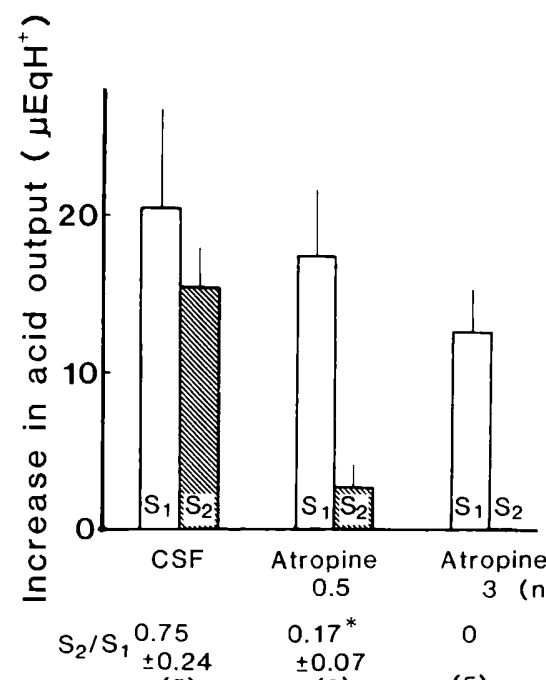

(5)

(3)
(5)

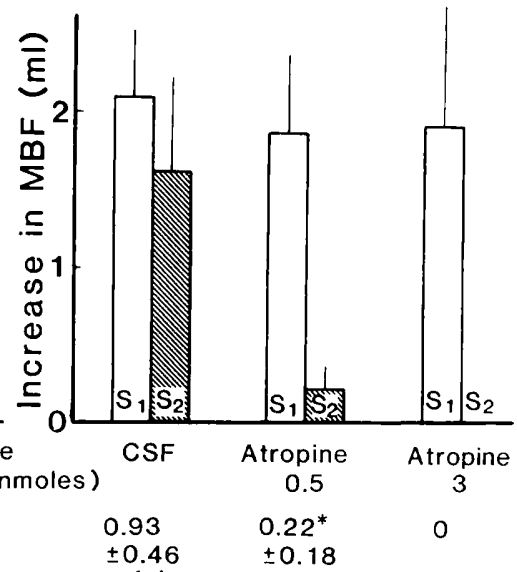

(5)

(5)

* $: \mathrm{P}<0.05$

Fig. 4. Effect of atropine intraventricularly applied on the increases in gastric acid output and MBF induced by electrical stimulation of the LHA. The results were expressed as the net increases in gastric parameters by the stimulations. $S_{1}$ and $S_{2}$ represent the values by the first and second stimulations, respectively, and the effect of atropine was evaluated as the ratio $S_{2} / S_{1}$. Test substances were applied intraventricularly $15 \mathrm{~min}$ prior to the second stimulation. ${ }^{*} \mathrm{P}<0.05,{ }^{*} \mathrm{P}<0.01$ (statistically significant difference from the respective control value). The number of experiments is given in parenthesis. 
Unilateral electrical stimulation of the LHA elicited consistent increases in gastric acid output and MBF. These increases were reproducible and were not significantly affected by intraventricularly applied artificial cerebrospinal fluid $15 \mathrm{~min}$ prior to the second stimulation (Figs. 3 and 4 ).

Intraventricular administration of atropine 1 and 5 nmole $15 \mathrm{~min}$ prior to the second electrical stimulation, dose-dependently inhibited the stimulation-induced increases in gastric acid output and MBF (Fig. 4).

Effect of atropine microinjected into the dorsal vagal complex on increases in gastric acid output and MBF induced by electrical stimulation of the LHA and intravenous administration of 2-DG: Pretreatment of rats with $0.5-3$ nmole of atropine microinjected into the ipsilateral dorsal vagal complex inhibited the increase in both gastric acid output and MBF induced by electrical stimulation of the LHA (Fig. 5). The degree of inhibition by 0.5 nmole of atropine corresponded well with that by 5 nmole of this agent intraventricularly applied. On the other hand, atropine in a dose of 0.5 nmole intravenously administered did not significantly affect the increase in acid secretion induced by stimulation of the LHA (data not shown).

Intranvenous administration of $60 \mathrm{mg} / \mathrm{kg}$ 2-DG induced a marked and long-lasting increase in both gastric acid output and MBF. Acid output at the 60 min collection period after administration of $2-D G$ in the control group reached $40.1 \pm 3.1 \mu \mathrm{Eq} / 15 \mathrm{~min}$ $(n=25)$, and that of MBF reached $6.91 \pm 0.58$ $\mathrm{ml} / 15 \mathrm{~min}(\mathrm{n}=25)$. Because of the relatively large individual variations, the results obtained after the administration of test substances were expressed as a percentage of the respective levels at $60 \mathrm{~min}$ after 2-DG. Atropine, 0.5-3 nmole, microiniected into the dorsal vagal complex bilaterally induced a dose-dependent and significant inhibition of the 2-DG-induced increases in gastric acid secretion and MBF (Fig. 6).

\section{Discussion}

In the present study, bethanechol but not nicotine microinjected into the dorsal vagal complex induced an increase in both gastric acid output and MBF.

It was, therefore, considered that central cholinergic muscarinic mechanisms exist within the dorsal vagal complex for excitation of gastric functions.

According to histochemical studies, direct

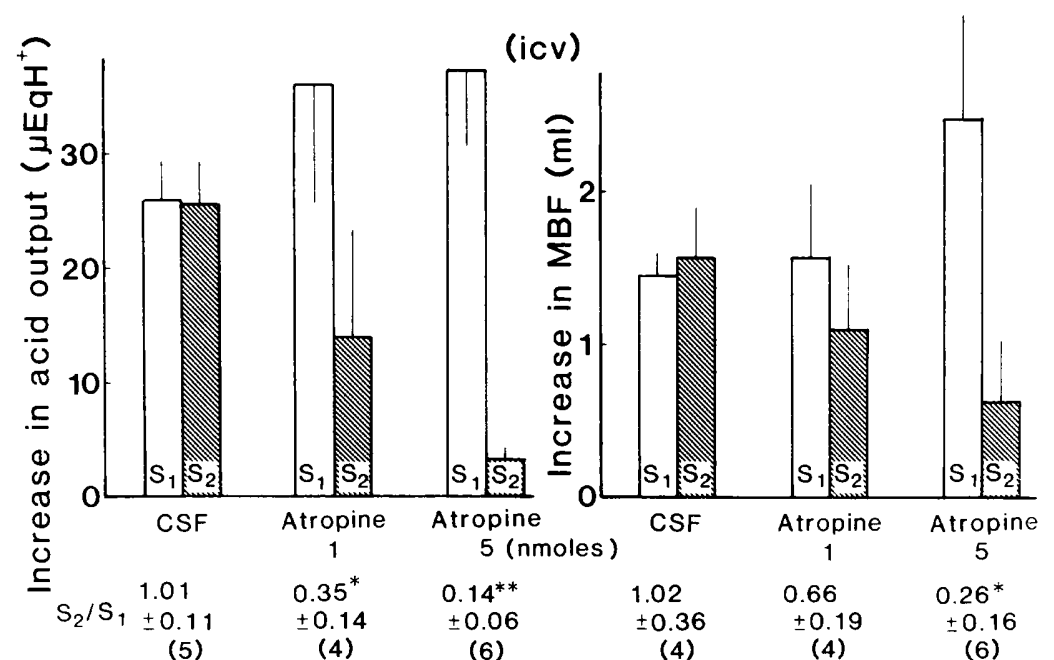

Fig. 5. Effect of atropine microinjected into the ipsilateral dorsal vagal complex on the increases in gastric acid output and MBF induced by electrical stimulation of the LHA. Test substances were microinjected into the ipsilateral dorsal vagal complex $15 \mathrm{~min}$ prior to the second stimulation. Other experimental procedures were described in the legend to Fig. 4. 

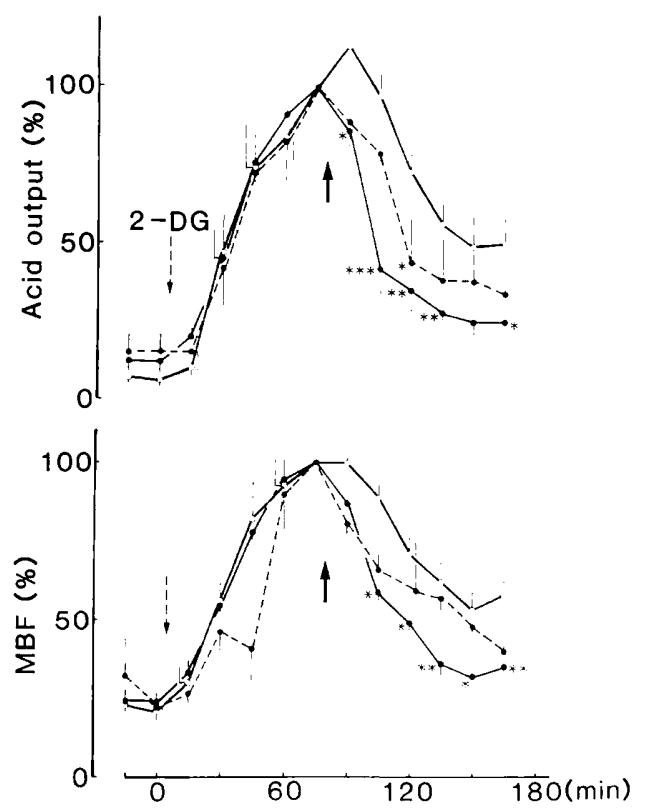

Fig. 6. Effect of atropine microinjected into the dorsal vagal complex on the 2-DG-induced increases in gastric acid output and MBF. A half amount of the respective dose was given into the dorsal vagal complex in a volume of $0.5 \mu$ each. $\hat{\imath}$ : Administration of test substances into the dorsal vagal complex. $\bigcirc-O$ : saline $(n=10)$,

0.5 nmole atropine $(n=6), 0-3$ nmole atropine $(n=9)$. ${ }^{*} P<0.05,{ }^{*} P<0.01,{ }^{* *} P<0.001$ (statistically significant difference from the control with saline).

descending hypothalamus-NDV fiber connections originating from the paraventricular nucleus, the LHA and the other regions have been demonstrated $(26,27)$. The presence of indirect polysynaptic fiber connections between the hypothalamus and NDV was also reported (28). However, descending fiber pathways between the LHA and NDV involved in excitatory regulation of gastric functions have not been identified.

Increase in gastric acid output induced by activation of the LHA neuron (2) can be explained by activation of motor pathways originating from the LHA and descending to the brain stem NDV (6). In the present study, both intraventricularly applied atropine and atropine microinjected into the dorsal vagal complex inhibited the increases in gastric acid output and MBF induced by electrical stimulation of the LHA. Furthermore, the increases in these gastric parameters induced by the administration of 2-DG were also significantly inhibited by atropine microinjected into the dorsal vagal complex. The principal site of action of 2-DG seems to be the chemoreceptor cells within the LHA (29).

The present study, therefore, demonstrates that the neurotransmission of the LHA-NDV descending pathways in regulation of gastric functions is probably mediated through cholinergic muscarinic receptors, at the level of the NDV. However, a possibility that the site of action of test substances microinjected into the dorsal vagal complex was the nucleus tractus solitarius but not the NDV can not be ruled out, since the NDV and the nucleus tractus solitarius are in close proximity, and there exists a functional circuit between these two nuclei.

Acknowledgements: This work was supported by Grants-in Aid for Scientific Research, Nos. 577109 and 5622102, from the Ministry of Education, Science and Culture, Japan, and by a Grant from the Japan Tobacco and Salt Public Corporation.

\section{References}

1 Mason, G.R. and Nelsen, T.S.: Gastric secretory and motor responses to anterior hypothalamic stimulation. Am. J. Physiol. 217, 1771-1775 (1969)

2 Misher, A. and Brooks, F.P.: Electrical stimulation of hypothalamus and gastric secretion in the albino rat. Am. J. Physiol. 211, 403-406 (1966)

3 Porter, R.W., Movius, H.J. and French, J.D.: Hypothalamic influences on hydrochloric acid secretion of the stomach. Surgery 33, 875-880 (1953)

4 Ridley, P.T. and Brooks, F.P.: Alterations in gastric secretion following hypothalamic lesions producing hyperphagia. Am. J. Physiol. 209, 319-323 (1965)

5 Kadekaro, M., Timo-laria, C. and Vincentini, M.L.M.: Gastric secretion provoked by functional cytoglucopenia in the nuclei of the solitary tract in the cat. J. Physiol. (Lond.) 299, 397-407 (1980)

6 Kerr, F.W.L. and Preshaw, R.M.: Secretomotor function of the dorsal motor nucleus of the vagus. J. Physiol. (Lond.) 205, 405-415 (1969)

7 Okuma, Y., Osumi, Y., Ishikawa, T. and Nagata, M.: Central noradrenergic-cholinergic interaction in regulation of gastric acid secretion in rats. Life 
Sci. 32, 1363-1370 (1983)

8 Osumi, Y., Aibara, S., Sakae, K. and Fujiwara, M.: Central noradrenergic inhibition of gastric muosal blood flow and acid secretion in rats. Life Sci. 20, 1407-1416 (1977)

9 Osumi, Y., Ishikawa, T., Okuma, Y., Nagasaka, Y.

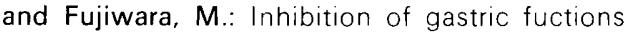
by stimulation of the rat locus coeruleus. Eur. J. Pharmacol. 75, 27-35 (1981)

10 Anichkov, S.V. and Grechishkin, L.L.: Influence of acetylcholine and benactyzine on central cholinergic structures regulating gastric secretion. Arch. Int. Pharmacodyn. Ther. 166, 417-423 (1967)

11 Emmerin, N. and Kahlson, G.S.: Histamine as a physiological excitant of acid gastric secretion. Acta Physiol. Scand. 8, 289-304 (1944)

12 Zawoiski, E.J. and Koplovitz, I.: Gastric secretory response of the anesthetized dog after direct chemical stimulation of the supraoptic region. Exp. Neurol. 55, 122-132 (1977)

13 Zawoiski, E.J.: The central activity of parasympathomimetics of gastric secretory function. Exp. Neurol. 34, 355-371 (1972)

14 Duke, W.W., Hirschowitz, B.I. and Sachs, G.: Vagal stimulation of gastric secretion in man by 2-deoxy-D-glucose. Lancet ii, 871-876 (1965)

15 Hirschowitz, B.I. and Sachs, G.: Vagal gastric secretory stimulation by 2-deoxy-D-glucose. Am. J. Physiol. 209, 452-460 (1965)

16 Taché, Y., Vale, W. and Brown, M.: Thyrotropinreleasing hormone-CNS action to stimulate gastric acid secretion. Nature 287, 149-151 (1980)

17 Ishikawa, T., Osumi, Y. and Nakagawa, T.: Cholecystokinin intracerebroventricularly applied stimulates gastric acid secretion. Brain Res. 333, 197-199 (1985)

18 Main, I.H.M. and Whittle, B.J.R.: Gastric mucosal blood flow during pentagastrin- and histamine-stimulated acid secretion in the rats. Br. J. Pharmacol. 49, 534-542 (1973)

19 Blair, E.L., Grund, E.R., Reed, J.D., Sanders, D.J., Sanger, G. and Shaw, B.: The effect of sympathetic nerve stimulation on serum gastrin. gastric acid secretion and mucosal blood flow responses to meat extract stimulation in anesthetized cats. J. Physiol. (Lond.) 253, 493-504 (1975)

20 Jacobson, E.D., Linford, R.H. and Grossman, M.I.: Gastric secretion in relation to mucosal blood flow studied by a clearance technic. J. Clin. Invest. 45, 1-13 (1966)

21 Shore, P.A., Brodie, B.B. and Hogben, C.A.M.: The gastric secretion of drugs: a $\mathrm{pH}$ partition hypothesis. J. Pharmacol. Exp. Ther. 119, 361369 (1957)

22 Brodie, B.B. and Axelrod, J.: The fate of aminopyrine (pyramidon) in man and methods for the estimation of aminopyrine and its metabolites in biological material. J. Pharmacol. Exp. Ther. 99, 171-184 (1950)

23 König, J.F.R. and Klippel, R.A.: A Stereotaxic Atlas of the Forebrain and Lower Parts of the Brain. The Rat Brain. Williams and Wilkins, Baltimore (1963)

24 Falcon, J.C., II, Phillips, M.I., Hoffman, W.E. and Brody, M.J.: Effects of intraventricular angiotensin II mediated by the sympathetic nervous system. Am. J. Physiol. 235, H392H399 (1978)

25 Ishikawa, T., Osumi, Y., Fujiwara, M. and Nagata, M.: Possible roles of central cholinergic nicotinic mechanisms in regulation of gastric functions. Eur. J. Pharmacol. 80, 331-336 (1982)

26 Hosoya, Y. and Matsushita, M.: Brainstem projections from the lateral hypothalamic area in the rat, as studied with autoradiography. Neurosci. Lett. 24, 111-116 (1981)

27 Saper, C.B., Loewy, A.D., Swanson, L.W. and Cowan, W.M.: Direct hypothalamo-autonomic connections. Brain Res. 117, 305-312 (1976)

28 Horst, G.J. ter, Luiten, P.G.M. and Kuipers, F.: Descending pathways from hypothalamus to dorsal motor vagus and ambiguns nuclei in the rat. J. Auton. Nerv. Syst. 11, 59-75 (1984)

29 Colin-Jones, D.G. and Himsworth, R.L.: The location of the chemoreceptor controlling gastric acid secretion during hypoglycaemia. J. Physiol. (Lond.) 206, 397-409 (1970) 